\title{
$\frac{213}{11.9}$
}

\section{DWR- 1616}

\section{Category: UC-37}

$15-2389$

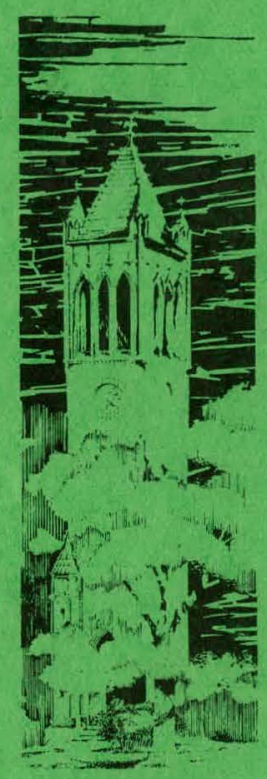

\section{IOWA STATE UNIVERSITY}

\author{
THE LOCK-IN DETECTOR
}

LOUIS MOURLAM, JR. AND WAYNE A. RHINEHART
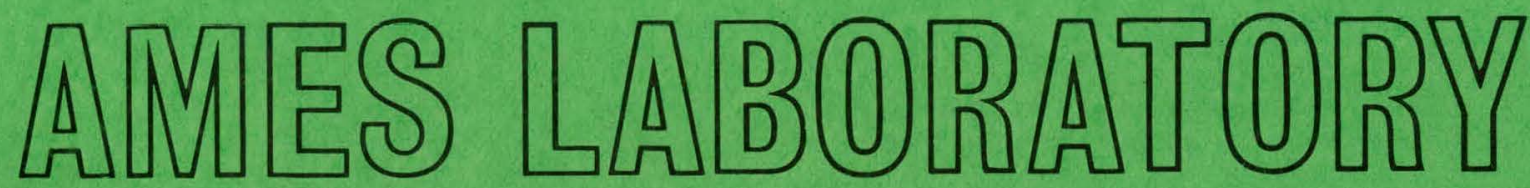

RESEARCH AND

DEVELOPMENT

REPORT

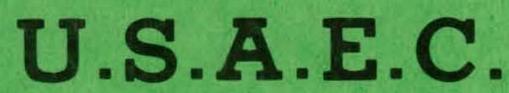

Date Published: September 1970

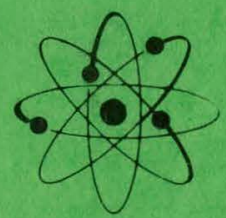




\section{DISCLAIMER}

This report was prepared as an account of work sponsored by an agency of the United States Government. Neither the United States Government nor any agency Thereof, nor any of their employees, makes any warranty, express or implied, or assumes any legal liability or responsibility for the accuracy, completeness, or usefulness of any information, apparatus, product, or process disclosed, or represents that its use would not infringe privately owned rights. Reference herein to any specific commercial product, process, or service by trade name, trademark, manufacturer, or otherwise does not necessarily constitute or imply its endorsement, recommendation, or favoring by the United States Government or any agency thereof. The views and opinions of authors expressed herein do not necessarily state or reflect those of the United States Government or any agency thereof. 


\section{DISCLAIMER}

Portions of this document may be illegible in electronic image products. Images are produced from the best available original document. 


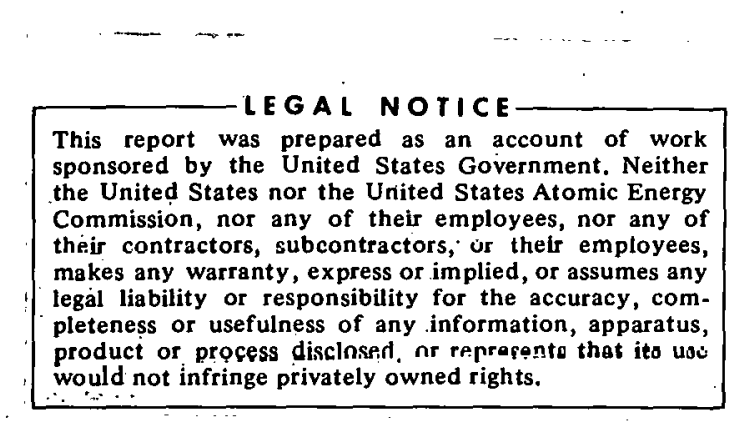

PREPARED FOR. THE U: $S$. ATOMIC ENERGY COMMISSION UNDER CONTRACT NO. W-7405-eng-82

Date Published: September 1970 


\section{LEGAL NOTICE}

This report was prepared as an account of Government sponsored work. Neither the United States, nor the Commission, nor any person acting on behalf of the Commission:

A. Makes any warranty or representation, expressed or implied, with respect to the accuracy, completeness, or usefulness. of the information contained in this report, or that the use of any information, apparatus, method, or process disclosed in this report may not infringe privately owned rights; or

B. Assumes any liabilities with respect to the use of, or for damages resulting from the use of any information, apparatus, method, or process disclosed in this report.

As used in the above, "person acting on behalf of the Commission" includes any employee or contractor of the Commission, or employee of such contractor, to the extent that such employee or contractor of the Commission, or employee of such contractor prepares, disseminates, or provides access to, any information pursuant to his employment or contract with the Commission, or his employment with such contractor.

Printed in the United States of America

Available from

Clearinghouse for Federal Scientific and Technical Information National Bureau of Standards, U.S. Department of Commerce Springfield, Virginia 22151

Price: Printed Copy $\$ 3.00$; Microfiche $\$ 0.65$ 
THE LOCK-IN DETECTOR

Louis Mour lam, Jr. and Wayne A. Rhinehart

Abstract

The fundamental principles involved in various lock-in detector systems are explained. Desirable features and shortcomings of each system technique are discussed. A procedure for evaluating the system most applicable to a particular experimental need is given. Basic operational problems which may occur in system usage are presented with suggested techniques for their solutions.

A circuit design for a dual phase lock-in detector system is given and explained in detail. This system is especially useful for balancing $A C$ bridges where the reactive and resistive components are being nulled simultaneously. Typical performance characteristics and operating data are listed. Helpful construction guides are given for the experimenter desiring to build this low cost, high performance detector. 


\section{PREFACE}

Scientists and engineers are often confronted with the problem of detecting the presence of low level signals which are to a large degree buried in White noise and/or $60 \mathrm{~Hz}$ pickup. The following sections deal with the theoretical and practical solutions involved in solving this common problem with an electronic device commonly known as the lock-in detector or sometimes referred to as the lock-in amplifier.

Section I deals with the basic questions concerning the lock-in detector which involves its description, operation, application, and what train of thought to use in evaluating the type needed.

Section 11 covers the most important factors to be considered when designing such an instrument. The analysis in this section is limited to the theoretical obstacles encountered when designing a lock-in detector and describes the basic design alternatives available at this time.

Section 111 describes the design and capabilities of a particular dual phase lock-in detector which can be constructed by the experimenter if its capabilities meet his requirements. Detailed operating instructions are included.

The objective of this report is to make well-known the fundamentals and subtle problems involved with lock-in detectors so that they can be used to the limit of their capabilities. 
TABLE OF CONTENTS

ABSTRACT

iii

I. FUNDAMENTALS OF THE LOCK-IN DETECTOR

What Is a Lock-In Detector?

How Does a Lock-In Detector System Work? -

Who Needs a Lock-in Detector? 7

Evaluating Lock-In Detector Systems _............ 8

Basic Operational Problems - 13

11. LOCK-IN DETECTOR SYSTEM DESIGN

AC Amplifier Design

Phase Sensitive Detector Design

Noise Rejection -

111. DUAL PHASE LOCK-IN DETECTOR

Basic Objective - 20

Over-All Approach - 22

Filling in the Blocks - 24

Amplifier Section - 24

Detector Section - 24

Power Supplies - 27

Construction

Operation

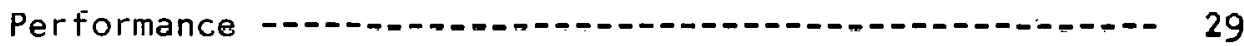

Operating Instructions - 30

IV. CONCLUSION 


\section{FUNDAMENTALS OF THE LOCK-IN DETECTOR}

The lock-in detector has in recent years emerged as a highly popular detection instrument in the field of scientific research. The technique has at times been claimed to be capable of solving all low level detection problems. As lock-in detectors have become available commercially, the advertising and specification writers have added a new dimension to the image of their product, one which does not always tell the entire story. The basic technique is by no means a recent technical development. Lock-in detection systems have been in use in various forms for over 25 years.

\section{What Is a Lock-In Detector?}

Commercial lock-in detectors and amplifiers have a great many features and operating controls which may cloud the basic function of the device. Basically they consist of an $A C$ amplifier followed by a phase sensitive circuit whose $D C$ output is proportional to (a) the input signal amplitude, and (b) the signal phase relative to an $A C$ reference voltage. The output of any $A C$ amplifier can be converted to DC either synchronously or asynchronously. A simple rectifier and filter would produce a DC output asynchronously; i.e., without regard to the phase of the signal. A lock-in detector produces a vi output synchronously, i.e., with regard to signal phase. The primary advantage of synchronous rectification is that signals of the same frequency as the desired signal but $90^{\circ}$ out of phase (in quadrature) can be completely rejected. It should be noted that infinite rejection occurs only at $90^{\circ}$ while other phases are passed with varying attenuation. Figure $l$ shows a $V_{\text {out }} v s$ phase plot which is typical of most lock-in detector systems.

Synchronous detection also has an advantage over asynchronous detection relative to noise rejection capability. An asynchronous system has no chance 


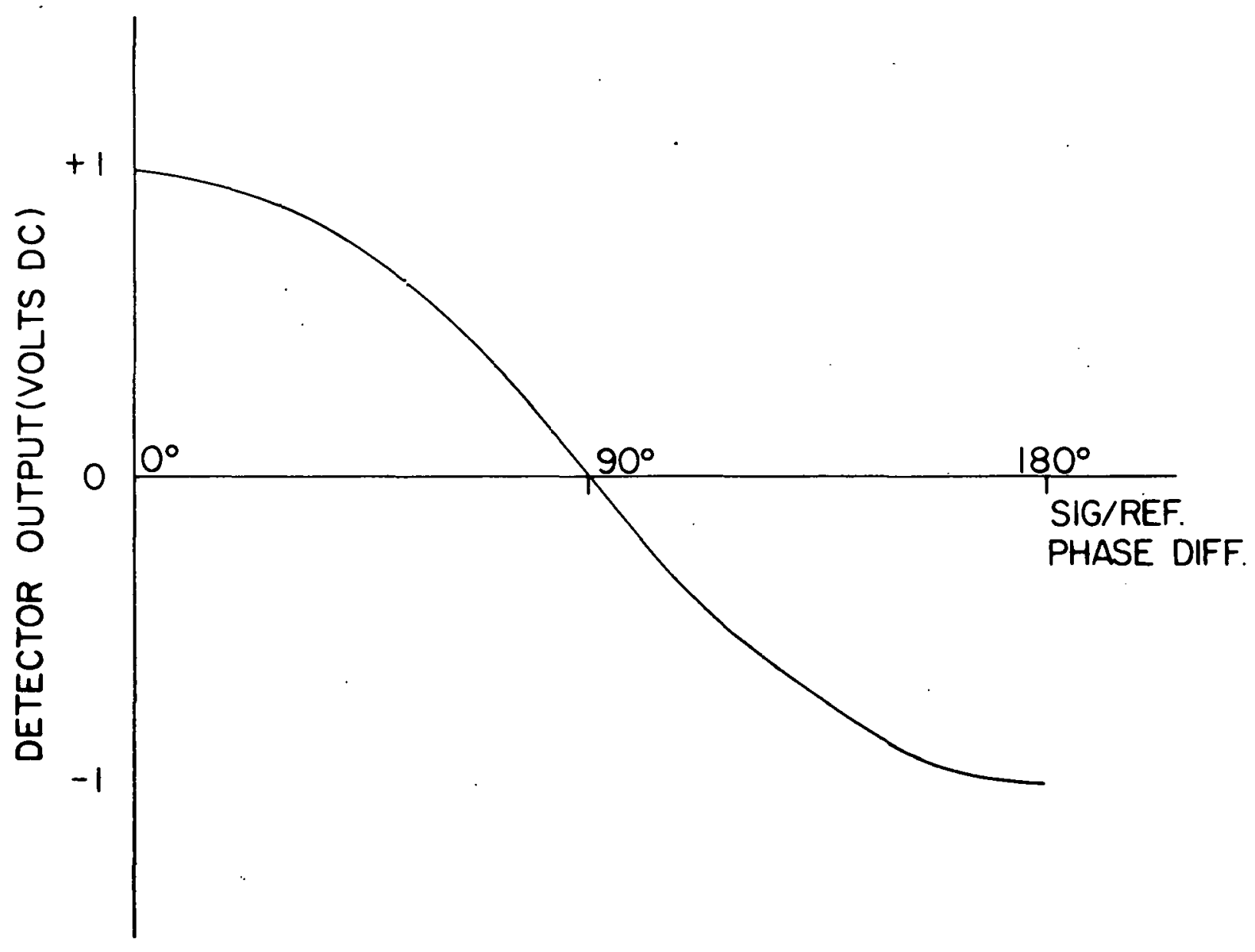

Fig. I. Vout vs phase. 
of rejecting noise which is within. the pass band of the instrument. A synchronous system can attenuate noise that lies in the pass band due to its phase coherent characteristic. It should be understood, however, that an uncorrelated signal is not completely rejected by a lock-in detector. The DC output due to a constant amplitude uncorrelated signal will vary randomly from a positive maximum through zero to a negative maximum. Johnson noise of source resistances and active amplifying elements which lies within the pass band of the lock-in detector is completely uncorrelated and will therefore produce a varying DC output as it randomly changes phase with respect to the lock-in detector reference voltage.

\section{How Does a Lock-In Detector System Work?}

The basic function of a lock-in detector system is the same as that of any $A C$ amplifier, i.e., to amplify an $A C$ voltage and provide an output suitable for displaying on a meter or recording device. The major operational difference is that the signal source must provide two inputs to the lock-in detector instead of one. In addition to the normal signal input from whatever phenomena are being observed, the signal source must supply the lock-in detector with an $A C$ reference voltage. If the signal source is an audio oscillator, the reference voltage is simply taken off in parallel with the signal driving the experimental bridge or whatever apparatus is being used.

A typical system using a lock-in detector would be as shown in Fig. 2 . A complete detection system consists of an oscillator and a lock-in detector. The oscillator drives the experimental apparatus which provides the electrical signal that is related to the phenomena being observed. The output signal, which is usually extremely low level, is entered at the signal input terminals of the system amplifier via a well-shielded, step-up transformer. The choice of 


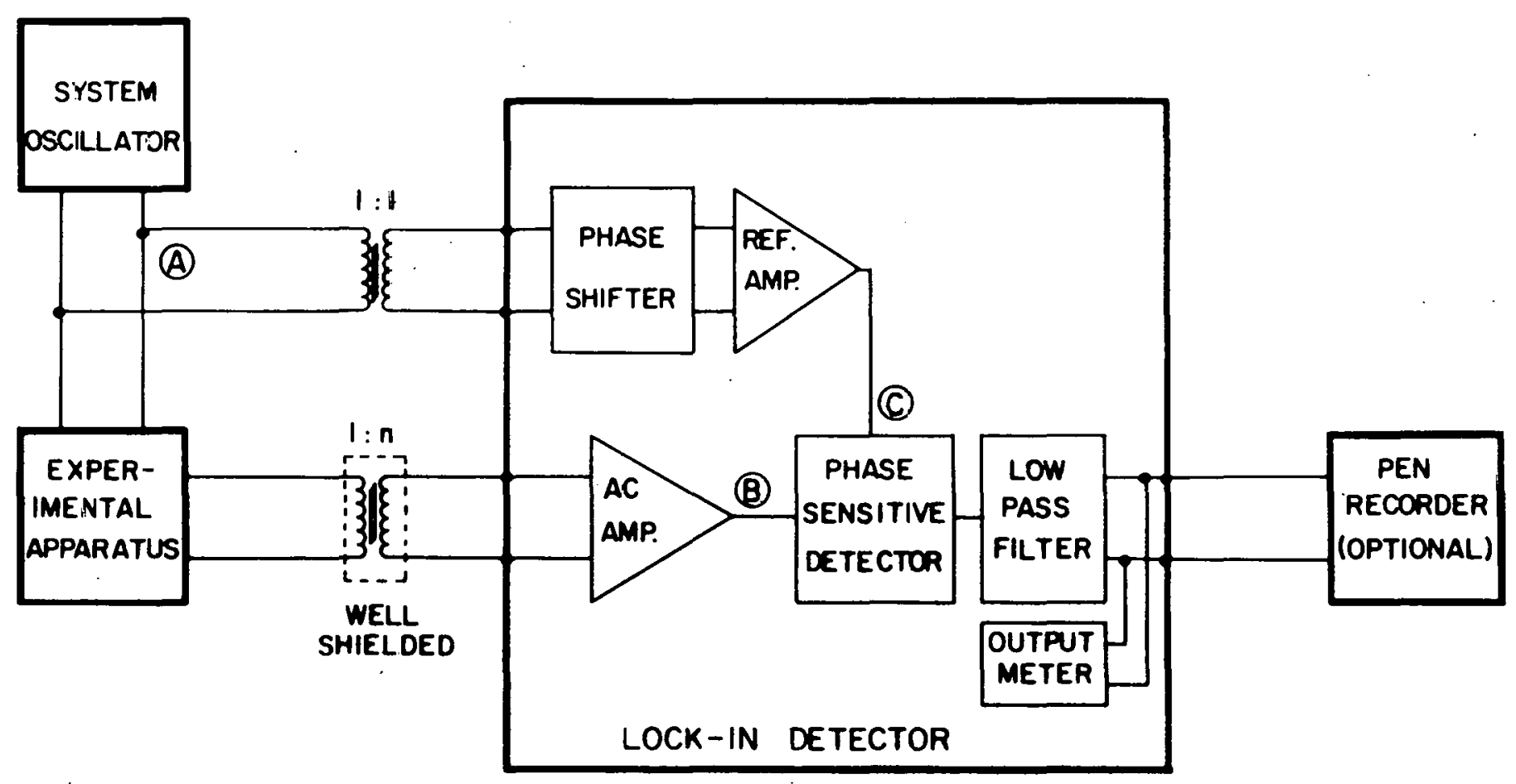

Fig. 2. Typical lock-in detector system. 
this transformer is critical to achieving maximum detection sensitivity. ${ }^{\dagger}$ The input signal passes through a narrow band amplifier which raises the desired signal to a level sufficient for the proper operation of the phase-sensitive detector. This is where the phase comparison of the amplifier input signal and the reference signal takes place. The unfiltered output of the detector will take many different shapes depending on the phase difference between the signal voltage and the reference voltage. A $0^{\circ}$ difference would give rise to a positive rectified output while a $180^{\circ}$ difference would produce a negative rectified output. Complex waveforms are produced for all phase differences other than $0^{\circ}$ and $180^{\circ}$. A variable time-constant, low-pass filter follows the detector. This filter determines the over-all bandwidth of the lock-in detector system and thus the noise rejection capabilities.

The important part of the reference channel is the variable phase shift control. This allows the operator to "lock-on" to the desired signal. The "lock-on" condition occurs when the signal and reference have a $0^{\circ}$ phase difference at the detector. This produces maximum $D C$ output for a given $A C$ input as shown in Fig. 1. Since the signal and reference both originate from the same place, namely point A in Fig. 2, they would be phase locked at the detector, points $B$ and $C$, if there were no other phase shifts present. However, there are always fixed phase shifts present in all experimental apparatus and in the amplifier section. The phase shift control is provided in the reference channel to compensate for whatever phase difference exists between the signal at point $B$ and the reference at point $C$. The lock-in detector is phased simply by adjusting the phase control for maximum DC output

+ W. A. Rhinehart and L. Mourlam, Elcctronies, Juhe 13, 1966, p. 114. 
on the front panel meter. After the lock-in detector, system is properly frequency tuned and phased, the front panel meter responds directly to changes in amplitude of the input signal whose phase is that of the original oscillator signal at point $A$. This is an important feature because any particular application, such as an $A C$ bridge, may produce an additional, often unwanted signal which is $90^{\circ}$ out of phase with the desired signal. A nonsynchronous system would have no way of separating the two signals, while the synchronous lock-in detector would reject completely the unwanted quadrature signal. This coherent detection characteristic is the most distinguishing feature of the lock-in detector system.

A second important feature of the lock-in detector is its ability to provide extremely high effective circuit $Q$ without the need for a highly tuned $A C$ amplifier. This is an important feature because it allows the use of a high $Q$ circuit without the need for an ultrastable system oscillator. In a highly tuned $A C$ amplifier, the tuned circuits determine both the bandwidth and the center frequency. In a lock-in detector system, the center frequency of operation is determined by the frequency of the reference voltage and the overall bandwidth is determined by the time constant of the low pass filter circuit in the detector output. Thus the high $Q$ is achieved at a point where the signal has been converted to a proportional DC voltage so that the stability of the oscillator is not a critical factor. The lock-in detector basically selects a bandwidth about the reference frequency and converts the signal information contained therein to a bandwidth starting at zero frequency (DC) and extending as high as desired. Since the bandwidth from DC up determines the over-all bandwidth, the $A C$ amplifier section can be relatively broad. This reduces the frequency stability requirements of the oscillator. If the $A C$ amplifier response is sufficiently broad, a frequency drift in the oscillator will not 
cause any appreciable change in over-all gain as would occur with a high $Q A C$ amplifier. This sums up the advantage of achieving high $Q$ operation with a lock-in detector as opposed to using a high $Q$ AC amplifier.

\section{Who Needs a Lock-In Detector?}

It is not possible to establish anything other than general guidelines for the application of the lock-in detector. The signal of interest must be made to appear at a single repetitive rate and the source must supply a reference voltage of the same frequency which is phase related to the signal. The lock-in detector is most useful in systems where extremely low level signals are of interest. The problem of recovering a nanovolt AC signal which is buried in noise is simply that of using a detecting device with a sufficiently narrow bandwidth to reject a majority of the noise thereby uncovering the desired signal. The classic noise vs time constant compromise applies, i.e., the narrower the detecting bandwidth (the larger the time constant), the better the noise rejection. The lock-in detector technique provides no magical solutions to this age-old conflict. The achievement of high effective $Q$ without the need for a critically tuned $A C$ amplifier is about the extent of the lock-in detector's advantage in this area. If low level signals are not involved, the only other major class of applications in which a lock-in detector is advantageous is where coherent detection is needed. Coherent detection is needed in situations where there is not only a signal due to the phenomena of interest but also an unwanted signal due to some other phenomena. If it is possible to code the wanted signal, e.g., chop it at a partlcular frequellcy, then the detector will decode the desired information while rejecting the unwanted signal. In situations of this type there is no alternative to a coherent detectiun systêli. 
A common lock-in detector application which involves the need for both low level detection and coherent operation is the detection of the null condition of an $A C$ bridge.

The output voltage of an unbalanced $A C$ bridge can be shown to contain two basic components: (1) the in-phase component which is nearly in phase with the oscillator driving the bridge, and (2) the quadrature component which is nearly $90^{\circ}$ out of phase with the bridge drive signal. The in-phase component is the result of the resistive unbalance of the bridge and the quadrature component is the result of the reactive unbalance of the bridge. A noncoherent detection system could not differentiate between the two. This would make the bridge balancing procedure quite complex and time consuming.

\section{Evaluating Lock-In Detector Systems}

A look at the specification sheet of a lock-in detector system reveals a long 1 ist of terms and numbers which range from easily understandable to incomprehensible. Translating what is needed for a specific task into a list for direct comparison is as improbable as completely understanding the specification sheet. Instead of playing the numbers game with the specification writers, the evaluation should, when possible, be based on a trial run under conditions at least similar to those of the final application. There are some basic factors which can be used to narrow the choice of a possibly suitable system.

The operating frequency can serve as one guide for sorting out the suitable from the unsuitable. Commercial lock-in detector systems can be equipped either with continuously tuneable frequency controls or with plug-in boards for fixed frequency operation. If the application requires changing the frequency of operation often, a tuneable model is indicated. If fixed frequency operation 
is sufficient, the plug-in models can greatly reduce the cost of the detection system.

The required input sensitivity may further reduce the potential candidates. To estimate the required maximum sensitivity, it is necessary to have at least. a rough idea of the minimum detectable signal of interest. The desired lock-in detector system should have a sensitivity range for which the minimum detectable signal is an appreciable part of full scale. If one wants to detect a few nanovolts, the lock-in system should have a full scale sensitivity of less than one microvolt available.

There is an operational subtlety of some lock-in detector systems which can preclude their use in some situations. Certain commercial models are built with an internal oscillator which can be used as the system oscillator. There are applications which call for the system oscillator to have exceptional amplitude stability. This could eliminate the use of the lock-in system's internal oscillator. A few lock-in detector systems are made which are not fully operable when used.with an external oscillator. This point should be verified before the final choice is made.

Another preliminary judgment to be made is the old stand-by, price. Commercial lock-in systems can cost anywhere from $\$ 900$ to $\$ 3,000$ so this subject is worth some study. The do-it-yourself approach should not be overlooked because, for many applications, a quite suitable lock-in detector system can be produced with about $\$ 200$ worth of parts and $50 \mathrm{~h}$ of a technician's time. This approach is certainly suitable for noncalibrated applications such as null detectors. In applications where absolute readings are taken, commercial lock-in detector systems with guaranteed calibration accuracy are probably the best choice. 
From these few considerations, it should be possible to move toward a final choice. Up to now the answers could be obtained either from commercial literature or from a sales representative. The remaining factors require having a lock-in detector system physically at hand for best results.

Probably the most aggravating point in all low-level detection systems is the problem of random noise. Before too much worry is expended about lockin detector system noise, the proposed system should be analyzed to see if detection sensitivity is actually affected by the lock-in detector system noise. This is dependent on the source of the signal which the lock-in system "sees." If the source random noise is far larger than the lock-in system noise, small differences in noise between different instruments is inconsequential.

The best way to objectively compare systems for internally generated random noise is with the setup shown in Fig. 3. This arrangement is designed to allow the calibration of the pen recorder so that zero and full scale on the recorder correspond with zero and full scale on the lock-in detector front panel meter. This should be done at a relatively high signal level, e.g., l mV, so that noise does not interfere. Noise comparisons must then be made at a single frequency because the internal lock-in system noise $r$ ises at lower frequencies due to the $1 / f$ noise phenomenon. After the full scale iecorder calibration is complete, the time constant switch setting must be set. The time constant calibrations on commercial detectors are not always directly comparable. The actual value should be determined since noise comparisons must be made at the same time constant. This is best accomplished by switching between a full scale signal and an input shorted condition while observing the time elapsed between $10 \%$ and $90 \%$ of full scale on the pen recorder. This assumes pen recorder response is much faster than the time constant being used. 


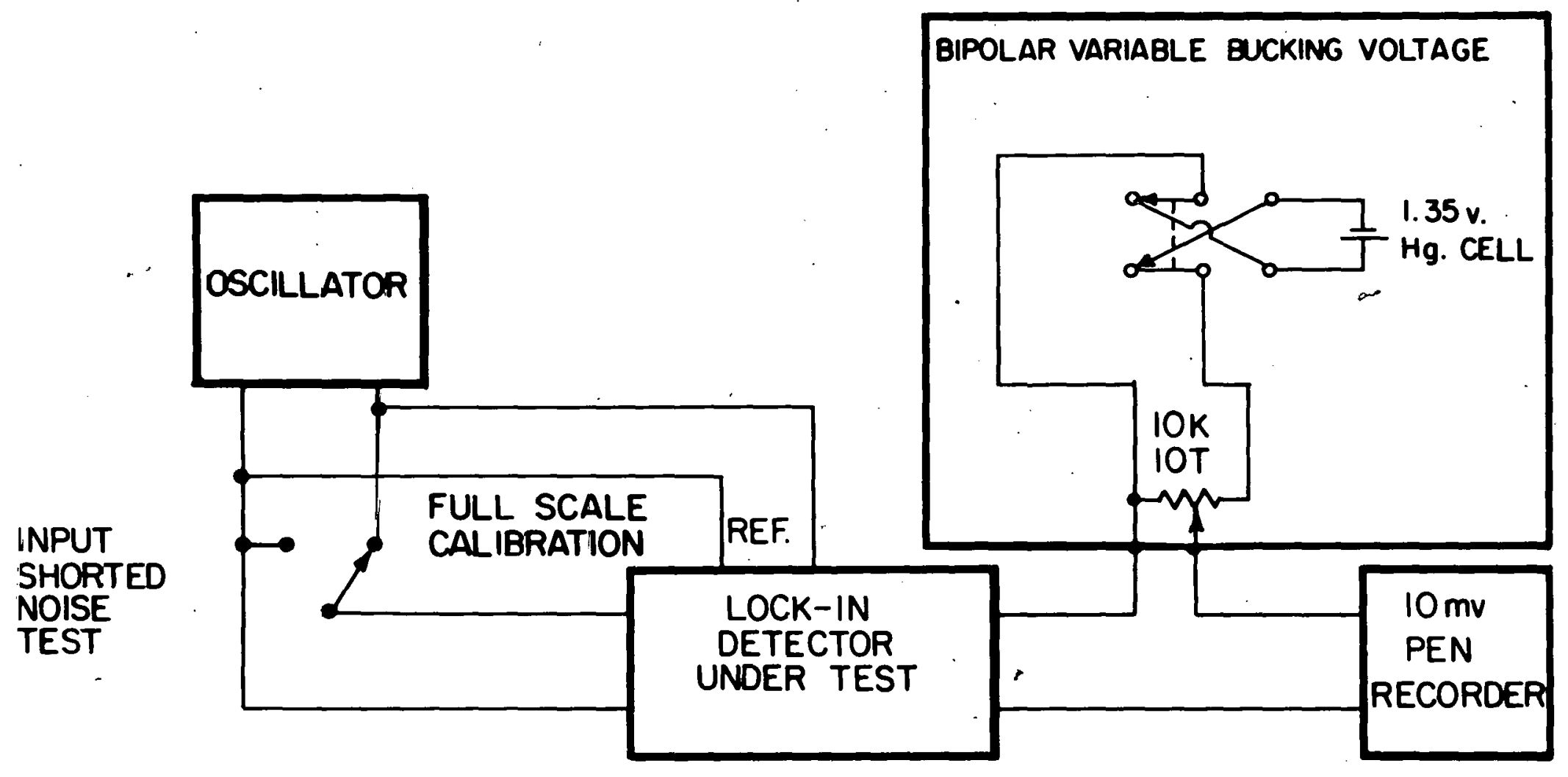

Fig. 3. Lock-in noise test setup. 
The choice of time constant for the comparison can be somewhere in the range of 1-3 sec when a recorder is used. The short-circuited input noise of most lock-in detector systems will fall somewhere in the range of 50-1000 nV@3 sec time constant depending on the frequency of test. The noise test should be made with the sensitivity control set for less than $1 \mu V$ full scale and the input short-circuited so that the noise provides a measurable band on the pen recorder. If the lock-in detector system under test does not go that high in sensitivity, expanded scale operation is necessary. This feature is built into many systems. If not, it simply involves a variable bucking voltage as shown in Fig. 3. For example, the lowest sensitivity setting might be $100 \mu \mathrm{V}$ full scale which might result in $1 \mathrm{~V}$ DC at the recorder output terminal. To make, the recorder sensitivity $\mathrm{I} \mu \mathrm{V}$ ( $1 \%$ of full scale) it is necessary to buck out $990 \mathrm{mV}$ ( $99 \%$ of full scale) and display the remaining $1 \%$ of full scale on a $10 \mathrm{mV}$ pen recorder. The resultant recorder trace should fluctuate an amount equal to a known number of nanovolts referred to the lock-in system input. Since the input is shorted, this fluctuation represents the noise generated internally in the system. Since most manufacturers use nuvistor tube input stages in their most sensitive models, the short-circuited input noise will usually not differ significantly from one lock-in to another among the more expensive systems.

Beyond the above mentioned considerations, additional evaluation of the lock-in detector systems will not usually uncover anything which would significantly affect the choice. Most have $360^{\circ}$ phase control, variable time constant controls, signal monitoring provisions, and built-in voltage calibrators. 
Basic Operational Problems

There are many operational difficulties peculiar to low signal level work which may be encountered when using a lock-in detector. They are often peculiar to a specific experiment and not general in nature. The few discussed here are limited to those which are most often encountered and apply to the lock-in's broad use.

The first point applies to the operation of a lock-in by a newcomer to this instrument. Such an operator can easily mistake the lock-in's random noise for an oscillatory condition when the amplifier section's output is viewed on an oscilloscope. With the input shorted and the sensitivity turned to maximum, the scope output will show a. sine wave whose amplitude varies randomly and can easily be interpreted as an oscillation since wideband random noise looks nothing like a sine wave. Since lock-ins are narrow band devices, however, they clearly support the Fourier analysis of a complex waveform by showing the operator one of the many sinusoidal frequencies which make up random noise. If such a signal is encountered with the lock-in sensitivity set at minimum, then an oscillatory condition may actually exist.

Physical placement of the lock-in in an experimental system can also cause problems. Whether any problems might be encountered depends highly on how well the amplifier section of the lock-in is magnetically shielded. The main adversaries are the system oscillator and $60 \mathrm{~Hz}$ broadcasters such as adjacent instruments which generate or consume high line power levels. If either causes problems, the lock-in's magnetic shielding must be improved or it must be physically separated from the sources of trouble mentioned.

Undoubtedly, the system problem most often encountered is the arrangement by which the experimental apparatus, the lock-in detector, a pen recorder, and the system oscillator are connected with respect to grounding. Unfortunately, 
there are few concrete rules that will guarantee the avoidance of grounding problems which generally take the form of a signal at the lock-in's input due to circulating ground return currents rather than the actual signal desired. In experiments where maximum sensitivity is desired grounding problems are most prevalent, and trial and error is the only avenue open to solve the problems that often are peculiar only to the specific experimental arrangement. It is generally best to earth-ground one side of the input to the lock-in which makes the most sensitive point least vulnerable to stray pickup signals. Interrupting the $A C$ line cord earth grounds on some or all of the instruments often breaks unapparent circulating ground paths. In general, it should be kept in mind that many experimental system problems can be attributed to the method of grounding used and the degree of familiarity with the lock-in detector.

\section{LOCK-IN DETECTOR SYSTEM DESIGN}

The lock-in detector system consists of a collection of ordinary circuits, i.e., amplifiers, phase detector, filters, etc. When they are applied to the lock-in detector, however, there are peculiarities which must be considered so as not to defeat the advantages that the technique possesses.

\section{AC Amplifier Design}

The design of the $A C$ signal amplifier section of a lock-in amplifier involves compromise among several divergent factors. This requires value judgment on the degree of compromise which can differ from one applicatiuil to another. The primary design questions which must be answered include: (a) How does one achieve the best low noise, high input impedance amplifier? and (b) What should 
the frequency response about the center frequency look like?

Significant random noise generated by an amplifier can be confined to the input stage if the first stage gain amplifies both signal and noise to a level significantly above the noise of succeeding stages. If a unity gain first stage is used, then the second stage noise also contributes directly to the amplifier's internally generated noise. Thus the resultant amplifier noise can be a function of the circuit configuration used. In general, it is best to minimize the number of active elements before the first stage of gain. The best that can be done is to make the very first active element provide the necessary gain to swamp out succeeding stage noise. A gain of ten would normally accomplish this objective. After the circuit configuration has been optimized, the remaining factor which will affect the over-all amplifier noise is the choice of the initial active element and its operating point. The choice of the active element for low noise undoubtedly could furnish hours of debate among the various component manufacturers. It appears that lock-in system designers are in general agreement on what device is best. The two prime candidates for providing high input impedance and low noise are the field effect transistor and the nuvistor vacuum tube. The field effect transistor has the advantage of no filament and essentially no microphonic noise problems. In the frequency range where lock-in detectors are most commonly used, i.e., below $1000 \mathrm{~Hz}$, the nuvistor tube generates less random noise. One particular direct comparison resulted in a nuvistor exhibiting a factor of two less noise than a field effect transistor when used in the same amplifier configuration. The advent of the low voltage nuvistor has allowed a hybrid nuvistor/transistor design which makes the use of a vacuum tube more palatable. The nuvistor is especially designed to reduce microphonic noise as compared to most vacuum tubes. 
The majority of lock-in system designers have settled on the nuvistor tube as the principal low noise amplifying device in the $A C$ amplifier section. After the initial stage, the amplification can take almost any form as far as random noise generation is concerned.

The question of the frequency response curve brings up more compromises. The main points in question are: (a) What is the optimum bandwidth for the $A C$ amplifier? and (b) What shape about the center frequency should be employed?

The bandwidth of the $A C$ amplifier should be relatively wide in applications where gain stability is important because a slight drift in the center frequency would cause a significant change in amplifier gain and phase shift if the circuit were highly tuned. Yet a narrow bandwidth is desirable because of its noise and $60 \mathrm{~Hz}$ rejection capability. Since the over-all lock-in system bandwidth is determined by the detector output filter, the $A C$ amplifier needs only to be narrow enough to prevent random noise and $60 \mathrm{~Hz}$ pickup prior to the amplifier input from overloading the amplifier itself and/or the phase sensitive detector. In applications where gain stability is not particularly important, such as null detection work, the amplifier can be narrower in bandwidth without loss of performance. These considerations have led to the inclusion of a variable $Q$ control on many commercial lock-in detector systems. The alternative to the variable $Q$ control is to design the amplifier with a fixed relatively low $Q$ but with an extraordinary dynamic range in both amplifier and detector so as not to risk overload. This approach cannot protect against all possible situations as there is a limit to the dynamic range that is obtainable with reasonable design and cost considerations.

The shape of the response curve about the center frequency can have importance in applications where gain stability is a factor. The Gaussian 
shaped response curve is attractive because it involves the most simplified circuitry but gives the least gain stability with frequency change since there is no appreciable flat region about the center frequency. A more ideal response is one that is rectangular in shape with an appreciable region about the center frequency where the amplifier gain and phase shift is relatively constant with frequency change. This form of response involves more complex circuitry which may not be practical at extremely low signal levels unless a special low level preamplifier is used. The preamplifier must then be extremely broad so as not to negate the advantages gained by the flat top response. This in turn makes the preamplifier vulnerable to overload in conditions where large random noise and $60 \mathrm{~Hz}$ signals accompany the signal of interest.

It can be concluded that applications where gain stability is needed and large noise components also exist are extremely difficult to cope with. In such situations, best results are obtained with a narrow bandwidth amplifier for noise rejection with the requirement of good system oscillator frequency stability. Commercial lock-in system manufacturers use the various techniques described and claim that signals 60-80 $\mathrm{AB}$ below the existing noise can be recovered. The majority of low level detection situations fall well within this specification.

\section{Phase Sensitive Detector Design}

of all the circuits which make up a lock-in detector system, the phase sensitive detector contributes most to giving the technique its unique characteristics: This being the case, it might be expected that the most stringent design considerations would apply to the detector circuit. Fortunately, there are dozens of circuit configurations which will perform this important function. The design of a suitable phase detector would depend on considerations such as 
linearity and overload characteristics which may be of concern to the user.

In general, they all have a $V_{\text {out }} v s$ phase plot similar to the one shown in Fig. 1. Phase detectors are also frequency sensitive as shown in Fig. 4. The even harmonics are completely rejected and the odd harmonics are passed without attenuation. This response emphasizes the need for a limited band pass amplifier ahead of the phase detector so as not to allow appreciable amounts of unwanted harmonic frequencies to reach the detector input. Therefore, a phase detector by itself cannot be considered a completely narrow band device.

The other primary points of interest are the dynamic range and the linearity of the detector circuit. The dynamic range is mainly involved with noise considerations. Since many lock-in systems use a moderately narrow band amplifier, there are noise components which reach the detector input and will not be removed until they reach the detector output low pass filter. The detector circuit should have sufficient dynamic range to pass these noise components and the desired signal without blocking or limiting. Linearity is a problem in situations where the lock-in detector is used as a calibrated voltmeter. Applications such as null detection do not require particular attention to linearity.

Noise Rejection

Noise rejection in lock-in detector systems is primarily the function of the $A C$ amplifier and the detector output low pass filter circuit. Both circuits accomplish the objective simply by being narrow band devices. Since lock-In systems often are used to measure small signals in the presence of large noise components, the rejection of the $A C$ amplifier is needed to prevent overload conditions in both the amplifier and phase detector. The noise which finally appears on the output indicator is determined by the bandwidth of the low pass 


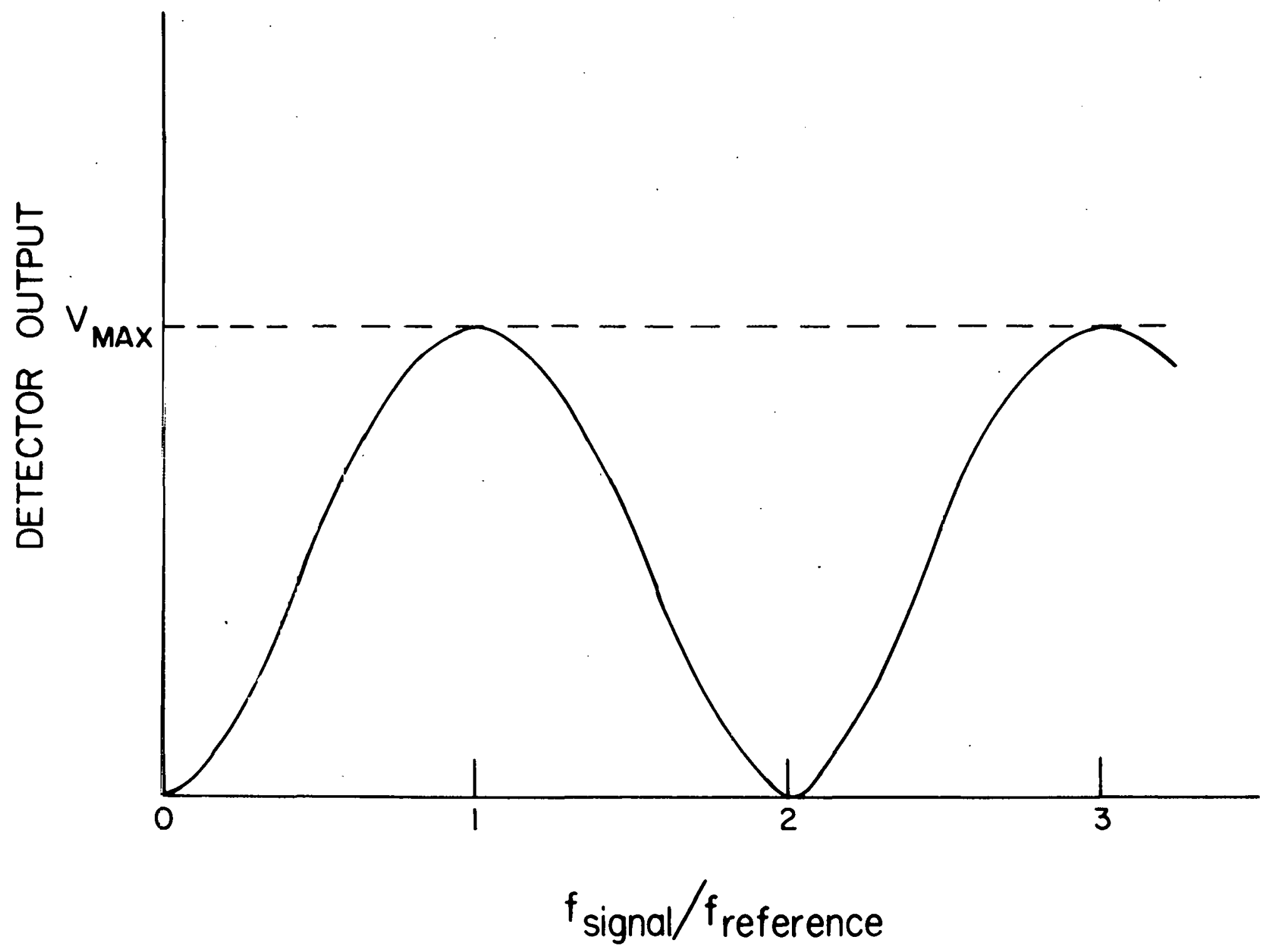

Fig. 4. Typical phase detector frequency response. 
filter circuit. The signal bandwidth about the reference frequency is converted by the phase detector to a detected band from DC up to any desired figure, say $10 \mathrm{~Hz}$. The output filter will pass any noise from the detector which lies within the signal bandwidth because that is where the useful information normally lies. Any unwanted high frequency components received from the detector will be removed by the low pass filter. The output of the detector may contain low frequency $A C$ signals which are a result of the signal frequency "beating" with some interference signal. Such low frequency signals can also be due to an interference signal which has amplitude modulated the signal frequency somewhere in the system. If these frequencies fall within the band pass of the filter, e.g., below the $10 \mathrm{~Hz}$ example given, there is no hope of removing them unless a narrower bandwidth output filter can be tolerated. This remedy is not always practical because the narrower the bandwidth, the slower the lock-in detector response becomes to a change in the input signal. For example, in null detection work a time constant $(10 \%-90 \%$ of full scale) of about $3 \mathrm{sec}$ is often the maximum which can be tolerated. The noise can be reduced to an arbitrarily small value by increasing the time constant (e noise $=k \sqrt{\Delta} f$ ). This is where the classic compromise of noise $v s$ bandwidlh makes its appearance.

\section{DUAL PHASE LOCK-IN DETECTOR}

\section{Basic Objective}

An application for which the lock-in detector is especially well suited is as a null detector in the precision balancing of an AC bridge circuit. This is an application for a low level device since the lower the bridge output is at "balance" the more accurate the results will be. It is also a situation 
where coherent detection is a necessity for best results because of the presence of the dual phase output of all AC bridges.

The so-called "in-phase" component of the bridge output, which is nearly in phase with the bridge driving oscillator signal, can be traced to the resistive unbalance of the bridge. The so-called "quadrature" component, which is nearly $90^{\circ}$ out of phase with the bridge oscillator signal, can be attributed to the reactive unbalance of the bridge. Thus an accurate bridge balance requires both a good resistive and reactive balance. The main difficulty in achieving this result lies in determining whether a given unbalance is due to the resistive component, the reactive component, or both. The lock-in detector allows one to "lock-on" to the in-phase component (thus rejecting the quadrature) and reduce it to a minimum with the resistive bridge control. After completion of the resistive balance, the lock-in detector can then be adjusted to "lock-on" to the quadrature component (while rejecting the in-phase) and reduce it to a minimum. Unfortunately, this cannot normally be accomplished in the two steps described. The component being rejected passes through the lock-in system's AC amplifier before it is discarded. Normally as one proceeds toward a balance condition, the detector gain is progressively increased as the component being balanced becomes smaller; however, since the rejected component stays substantially constant, a point will be reached when it will saturate the $A C$ amplifler and prevent further balancing of the bridge. This is due to the finite dynamic range of all AC amplifiers. Thus one must progress toward balance with both components, alternately locking onto one and then the other. An added complication often is encountered when the in-phase and quadrature bridge controls cannot be made entirely independent of each other. Even with the help of a phase coherent lockin detector, the bridge balancing process is quite lengthy and difficult. 
The quadrature rejection capability of the lock-in system's phase detector circuit suggests that a good solution to this dilemma exists. Since it is possible to "lock-on" to one component while completely rejecting the other, there should be no reason why a lock-in detector system could not have two phase detectors; one locked to the in-phase component and the other locked to the quadrature component. This would allow the operator to observe the condition of both the resistive and reactive unbalances simultaneously and make bridge adjustments accordingly without having to readjust the lock-in phase controls. Since each detector circuit has good rejection of signals $90^{\circ}$ out of phase with its reference signal, the in-phase component will not affect the quadrature detector and vice versa.

\section{Over-All Approach}

A design philosophy which is suitable for the achievement of a dual phase lock-in detector system is shown in the block diagram of Fig. 5. The signal input which accepts the AC bridge output consists of a narrow band, low noise $A C$ amplifier. As in the single phase lock-in, its function is to provide low noise gain sufficient to raise the signal to a detectable level, reject as much noise as possible, particularly. $60 \mathrm{~Hz}$, and provide a high input impedance.

The combination in-phase/quadrature signal, after being amplified, is ready to be separated into the two component parts for individual display. Since the phase sensitive detector circuit produces maximum output for only the phase of its reference, the in-phase detector is fed directly from point $A$ while the quadrature detector is fed through an additional phase control whlch allows an adjustment to place the quadrature detector response exactly $90^{\circ}$ (orthogonal) in relation to the in-phase detector. The initial phase control in the reference channel allows adjustment for locking the in-phase detector 


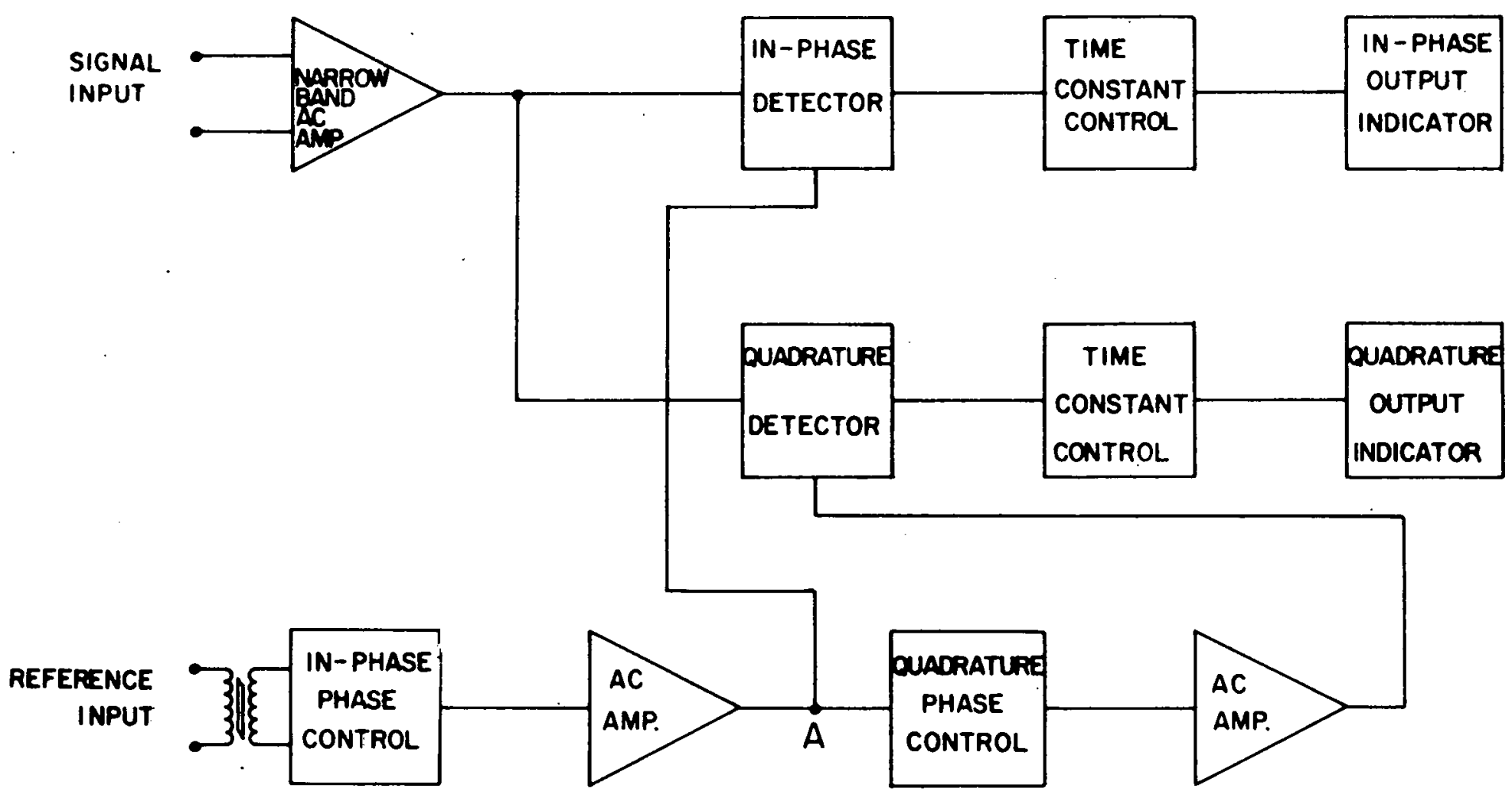

Fig. 5. Dual phase lock-in detector. 
to the in-phase signal. Thus when the in-phase detector is locked onto its signal, the quadrature detector can be adjusted to respond only to the quadrature signal from the bridge. When the phase controls are properly adjusted, the interaction between the two channels is insignificant. This eases the task of arriving at an accurate balance condition.

\section{Filling in the Blocks}

The circuits which occupy the blocks in Fig. 5 may vary in complexity depending upon the design criteria. A simplified dual phase lock-in detector system can be constructed by the do-it-yourself route in many cases. The circuit details which follow are best suited for the construction of a dual phase lock-in null detector.

\section{Amplifier Section}

The AC amplifier shown in Fig. 6 consists of two narrow band negative feedback loops each of which uses a balanced parallel tee filter circuit to achieve the limited band pass. The first feedback loop employs low voltage nuvistor tubes as the low noise initial amplifying element. It has a fixed $Q$ of about 100 with a forward signal gain of about 1200. The AC amplifier gain is adjustable by resistive attenuators at the input and between the two stages. The second stage is a broadly tuned parallel tee feedback amplifier which gives additional gain of about 1000 and more $60 \mathrm{~Hz}$ rejection.

\section{Detector Section}

The reference channels and detectors are shown in Fig. 7. The reference input, which requires a sinusoidal wave form, includes an isolation transformer to prevent ground loop conditions from disrupting normal lock-in detector operation. The reference circuitry consists basically of two identical 


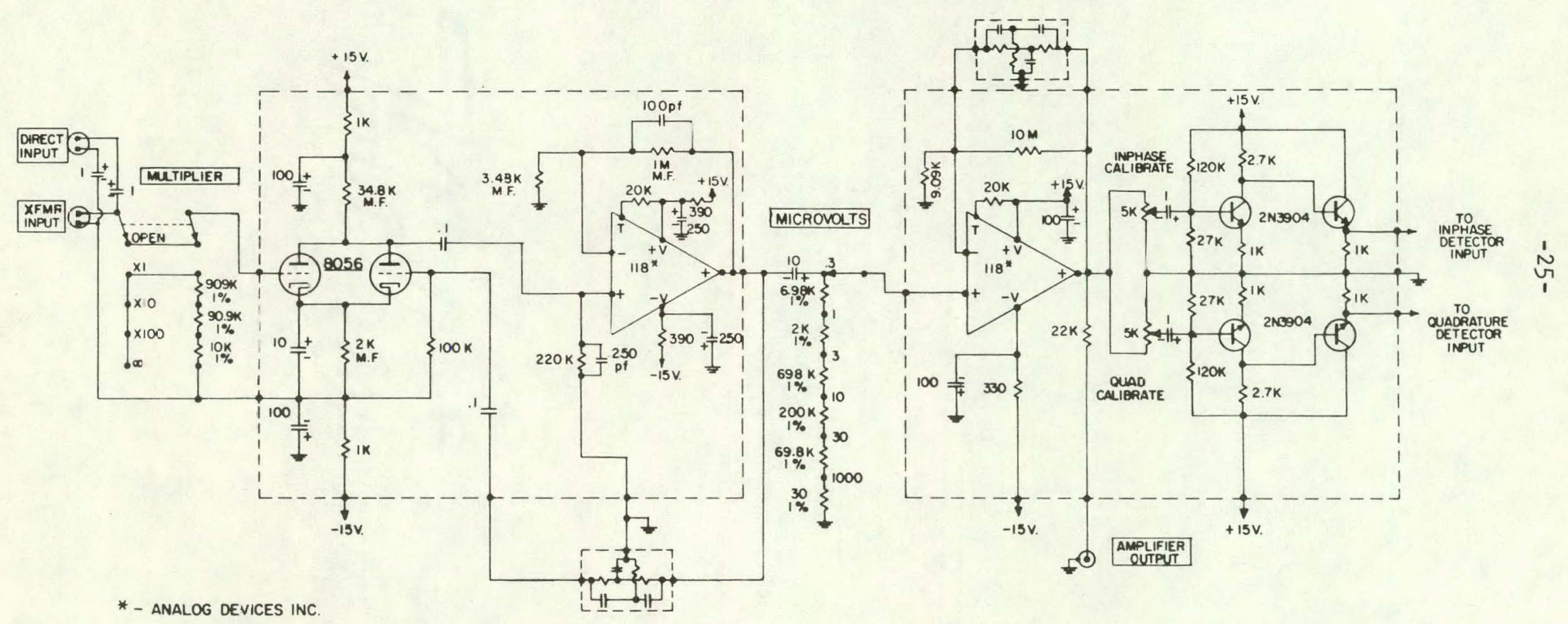

Fig. 6. Amplifier section. 


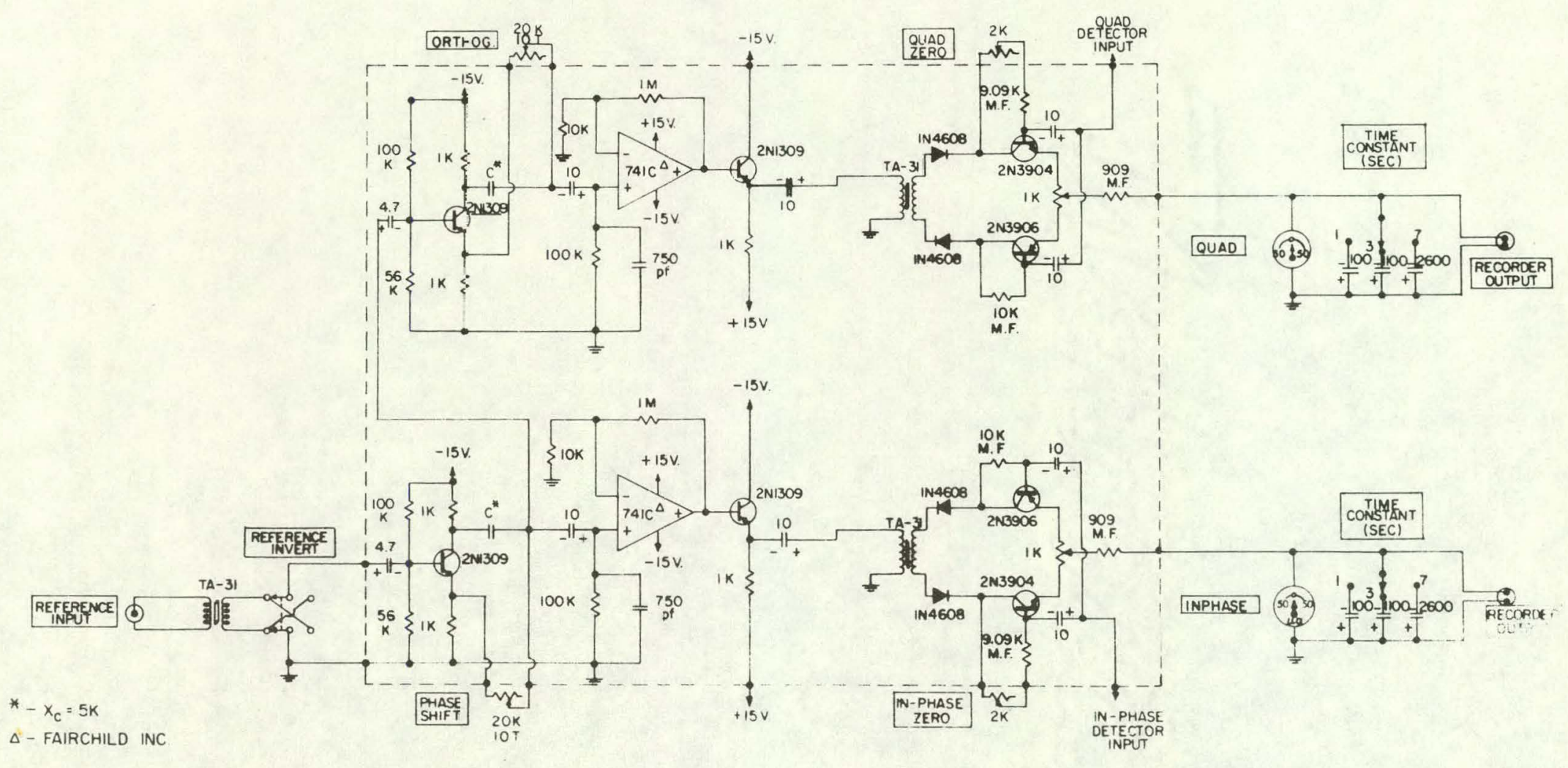


channels, one for the in-phase detector and one for the quadrature detector. The phase shift controls are followed by an integrated circuit operational amplifier which squares the reference voltage and provides immunity to changes in reference amplitude. The phase detector is a half wave type which drives the front panel meter directly. The detector output is then passed to a variable time constant filter circuit and to an output jack for display on a pen recorder.

\section{Power Supplies}

The power supplies used can be a source of trouble. They should be dual stage electronically regulated supplies as good low impedance supplies eliminate many potential problems of interaction between different sections of the lock-in system. It is best to use separate supplies for the amplifier section and the detector section to prevent coupling any of the high level reference signal into the low level signal circuits. This can be checked by observing the shortcircuited input noise at the amplifier output both with and without a reference signal. The noise level should remain constant if there. is no coupling problem.

\section{Construction}

Construction techniques used can be the source of many strange effects in do-it-yourself lock-in detector systems. The primary points to be considered are ground wire routing and proximity of sensitive circuits. Ground returns from low level circuits should not be common with returns from high level circuits. It is best not to allow a low level ground wire to lie adjacent to a high level ground wire. There should be no more than one point on the chassis which is tied to the circuit common. Separate ground wires from the different circuit cards in the lock-in should be used and brought to a single 
terminal to be grounded either to the chassis or to some external earth ground or system ground.

The high level reference circuits should be physically placed as far away from the low level amplifier input stage as possible. A few nanovolts of . reference voltage coupled into the signal input can cause oscillation or significantly raise the apparent noise output of the amplifier. It is best to be generous with shields between the circuit cards especially where the amplifier input is concerned. The input grid lead should be kept as short as possible and be well shielded.

\section{Operation}

The dual phase lock-in detector system is operated the same as a single phase type except for the added complexity of an additional phase control. The correct phasing of the lock-in detectors is very important in order to obtain best results. Improper phasing will generally result in excessive interaction between the in-phase and quadrature channels. The method of phasing is somewhat different than that normally used in a single phase unit. When first setting up a bridge balancing operation, the dual phase lock-in detector must be adjusted so that the in-phase and quadrature meters are responding only to their respective signals from the bridge. The precise lock-on point which will give maximum output is difficult to determine due to the relatively flat portion in this area of the response curve. However, about the $90^{\circ}$ (infinite rejection) point, the slope of the response curve is very steep and a small error. in the phase setting of the lock-on point will place the response to a $90^{\circ}$ signal significantly off the infinite rejection point. The result is interaction between the two channels. Therefore, finding the $90^{\circ}$ infinite rejection points of each chonncl is a more accurate means of phasing the detectors than phasing 
for a maximum desired output. Initially, a rough bridge balance can be obtained by observing the amplifier output on an oscilloscope and adjusting the bridge. components for a minimum signal condition. This step will not result in a particularly good bridge balance, but will enable one to proceed to the final precise setting of the phase controls. After the rough balance is completed, the reactive bridge control is adjusted for a large reactive unbalance and the PHASE control is adjusted for zero output (infinite rejection) on the in-phase meter. The ORTHOGONALITY control can be set similarly by causing a large resistive unbalance and adjusting the quadrature detector for infinite rejection. This approach results in the precise phasing of the lock-in detectors so that interaction between the in-phase and quadrature channels is insignficant. The need for a precision adjustment indicates that to maintain correct phasing the phase shifts present in the system should be constant and the frequency of the system oscillator must be reasonably stable. The former will be true if mica or mylar capacitors are used in the variable phase circuits and if the narrow band $A C$ amplifier is not too highly tuned. The oscillator frequency stability should be of the order of $\pm 0.01 \%$ to minimize drifts in phase settings.

\section{Performance}

The lock-in detector system described allows for the useful amplification of fractional nanovolt signals from low impedance sources. To be more specific, the short circuited input noise at $400 \mathrm{~Hz}$ with a $3-\mathrm{sec}$ time constant is typically $50 \mathrm{nV}$. An appropriate input transformer would allow the recovery of signals of. less than $1 \mathrm{nV}$ from a low impedance source. As described earlier, lock-in detector system performance is difficult to reduce to a few simple phrases. 


\section{Operating Instructions}

Phase Control Adjustment

Step 1: Allow amplifier and oscillator to warm up at least $1 \mathrm{~h}$ or until oscillator has achieved maximum stability.

Step 2: Adjust oscillator frequency to the center frequency of amplifier by observing for maximum SCOPE output. Set MULTIPLIER and/or MICROVOLT controls to keep both meters on scale throughout the lock-in's use.

Step 3: Perform a preliminary bridge balance using an oscilloscope attached to the SCOPE output as the indicator of the bridge's condition. I gnore meter indications during this step.

Step 4: Check zero setting of both meters by turning MULTIPLIER switch to infinity position. Adjust each meter ZERO if necessary.

Step 5: Increase MULTIPLIER by factor of at least 100. Unbalance bridge reactively until approximately full scale is read on QUADRATURE meter.

Step 6: Readjust front panel PHASE control for zero reading on IN-PHASE meter to maximize quadrature rejection. Rebalance bridge.

Orthogonality Control Adjustment

Step 1: Proceed through Step 6 as above if not previously done.

Step 2: Increase MULTIPLIEER by at least a factor of 100. Unbalance resistive component of bridge until approximately full scale is read on IN-PHASE meter.

Step 3: Adjust ORTHOGONALITY control for zero reading on QUADRATURE meter to maximize in-phase rejection. Proceed with normal bridge balance.

Further adjustment of either PHASE or ORTHOGONALITY controls should not be necessary as long as oscillator frequency remains constant. 


\section{CONCLUSION}

In conclusion, the user should note that the various circuit configurations and their complexity can result in several types of devices that are all referred to as "lock-in detector systems." The choice of the system which best suits a particular experimental need should be determined by comparing the relative merits and shortcomings of each system as applied to the specific problem under consideration. Unfortunately, there is no single system or device presently available which contains all the good features discussed and none of the shortcomings. It is hoped that the previous discussion will assist the experimenter in making the best choice for his own case. 